\title{
Agôn
}

Revue des arts de la scène

HS 2 | 2014

Mettre en scène le conte

\section{Hamlet et Cendrillon : deux orphelins face à la parole d'un parent mort}

\section{Delphine Urban}

\section{(2) OpenEdition}

\section{Journals}

\section{Édition électronique}

URL : http://journals.openedition.org/agon/3092

DOI : $10.4000 /$ agon.3092

ISSN : 1961-8581

\section{Éditeur}

Association Agôn

\section{Référence électronique}

Delphine Urban, « Hamlet et Cendrillon : deux orphelins face à la parole d'un parent mort », Agôn [En ligne], HS 2 | 2014, mis en ligne le 09 septembre 2014, consulté le 19 avril 2019. URL : http:// journals.openedition.org/agon/3092 ; DOI : 10.4000/agon.3092

Ce document a été généré automatiquement le 19 avril 2019

Association Agôn et les auteurs des articles 


\title{
Hamlet et Cendrillon : deux orphelins face à la parole d'un parent mort
}

\author{
Delphine Urban
}

1 Faire d'Hamlet une introduction à la représentation de Cendrillon? Réflexions autour d'un travail dramaturgique et scénique mené en 2013-2014 avec un groupe d'élèves de terminale Littéraire option obligatoire théâtre.

2 Les hasards des programmes (option obligatoire théâtre en terminale, programme limitatif pour l'année 2013-3014) font cette année se croiser deux jeunes gens : Hamlet, le jeune prince du Danemark en mal de père imaginé en 1601 par Shakespeare, et SandraCendrillon, la très jeune fille de la pièce de Joël Pommerat créée en 2011 aux ateliers Berthier. Les programmes n'incitent pas à particulièrement à commenter cette rencontre de circonstance. Au contraire, il s'agit plutôt d'explorer des domaines différents : une pièce élisabéthaine appartenant au patrimoine culturel et abondamment mise en scène, et la création de Pommerat, écrite et mise en scène par lui-même, qui relève de la démarche contemporaine dite d'« écriture de plateau » selon la proposition, discutée, de Bruno Tackels ${ }^{1}$. Pourtant, cette juxtaposition provisoire (l'an prochain, Hamlet ne sera plus au programme) ouvre des voies intéressantes pour l'interprétation des deux pièces. Avec ma classe de terminale, je me suis proposé cette année de les explorer en faisant d' Hamlet une sorte de prolégomènes à Cendrillon. Pommerat est un écrivain du palimpseste. Pour ne citer que deux exemples frappants: il revendique Au Monde comme sa propre version des Trois sours de Tchekhov et les parentés de Ma chambre froide avec La Bonne Âme $d u$ Setchouan ne sont plus à prouver. Shakespeare, moins évident, peut cependant être un autre hypotexte : ainsi, Au Monde est à certains points de vue une variation sur Le Roi Lear (le patriarche cédant son empire industriel non à ses trois filles, pourtant réunies, mais à son fils).

3 Dans Cendrillon, Pommerat s'inspire certes de Perrault dont il reprend le schéma narratif initial (une petite fille aux prises avec une marâtre injuste qu'on lui impose après la mort de sa mère) et auquel il adresse quelques clins d'œil de connivence (Sandra admirant les 
chaussures du prince au sortir du bal !) mais il déplace l'essentiel du conte dans une mise en évidence de la difficulté du deuil pour la très jeune fille qui vient de perdre sa mère. Les cendres dont la jeune souillon est barbouillée se font signes de mort, Sandra-Cendrier est une héroïne funéraire qui piétine dans le souvenir de sa mère comme Hamlet, dans la mise en scène d'Ostermeier (que les élèves ont vue en vidéo) s'englue dans la terre qui a enseveli son père. J'ai donc proposé aux élèves et à la comédienne-intervenante ${ }^{2}$ l'hypothèse que Cendrillon serait, dans la version de Pommerat, un avatar d'Hamlet. Notre travail sur le plateau s'est construit autour de ce motif.

\section{Hamlet et Cendrillon : drôle d'endroit pour une rencontre?}

Comme Hamlet qui vient de perdre son père, Sandra est privée du référent parental de son sexe. Comme Hamlet qui déteste son oncle, meurtrier potentiel, nouveau mari de sa mère, Cendrillon ne peut aimer une belle-mère qui la relègue à la cave pour ne pas avoir à entendre ses récurrentes références à sa mère. Dans les deux cas, le parent restant ne parvient pas à secourir son enfant, à le protéger des malveillances du beau-parent. On pourrait même dire qu'il ne le veut pas : Gertrude aurait pu dire à Hamlet ces paroles que son père dit à Sandra : « je suis encore jeune, je veux être heureux, j'ai envie de tourner la page, j'ai envie de refaire ma vie, de recommencer une nouvelle vie $»^{3}$. Avec la certitude de la jeunesse, l'orphelin condamne le parent veuf et refuse de se détacher du disparu, paré de toutes les vertus et pesant dans sa présence fantomatique. Hamlet est littéralement hanté par le spectre paternel qui lui apparait à deux reprises dans la pièce. Sandra fabrique son propre fantôme, matérialisé par divers accessoires, dont une montre ridicule qui, dans la mise en scène de Pommerat, fait régulièrement entendre les notes aigrelettes de la comptine «ah vous dirais-je maman ». C'est que, l'un comme l'autre, Hamlet comme Sandra, se croient, légitimement ou non, détenteur d'une parole posthume à laquelle leur existence doit être dévouée toute entière. Le spectre de son père ne s'adresse qu'au jeune Hamlet et clôt ses recommandations vengeresses par ce qui deviendra la devise du prince «Adieu, adieu, adieu, ne m'oublie pas »4. Quant à Sandra, appelée au chevet de sa mère mourante, elle croit aussi comprendre qu'une mission sacrée lui est confiée : «Maman, je te promets que je penserai à toi à chaque instant. J'ai très bien compris que c'est grâce à ça que tu mourras pas en vrai et que tu resteras en vie dans un endroit invisible tenu par des oiseaux. (...) Ne t'inquiète pas maman, je ne te laisserai pas mourir en vrai, tu peux compter sur moi. Tous les jours, à chaque minute, et pendant toute ma vie, tu seras dans mes pensées... $»^{5}$.

5 Il s'agit dans les deux cas d'un échange de mots lourd de conséquences: l'orphelin recueille une parole d'outre-tombe, ou presque, l'interprète seul, avec le filtre de sa souffrance, et donne au mort sa propre parole, un serment pour l'éternité qui engage toute son existence. Comme le résume l'une des élèves dans son carnet de bord: "Ces deux pièces construisent leurs intrigues sur le dernier message d'un parent défunt mal interprété par son enfant $»^{6}$. Les deux orphelins, persuadés d'être responsables du devenir de leur parent mort, s'interdisent alors de vivre, d'aimer, d'être beaux ou joyeux. Les pièces retracent ce complexe parcours du deuil qui conduira Hamlet à rejoindre son père et Sandra à renoncer à sa montre tonitruante. Parcours de mort pour l'un, de vie pour l'autre. A partir de ces constats, notre projet pour la partie pratique de l'épreuve du baccalauréat consiste à lier les deux œuvres, sans forcer sur des ressemblances grossies 
mais en en faisant entendre la proximité et la continuité. Le travail de pratique mené avec la comédienne Catherine Vuillez s'est attaché à explorer ce lien entre les deux pièces ${ }^{7}$. Le projet a été exposé d'emblée aux élèves qui en rendent compte dans leurs carnets de bord : « Notre projet débute avec la tragédie d'Hamlet de Shakespeare. Ce choix a été fait car un lien logique s'est offert à nous avec Cendrillon de Pommerat. Les deux pièces s'appuient sur le thème de leur défunt parent. S'il a été décidé de jouer Hamlet avant Cendrillon c'est dans le but de permettre une continuité entre les deux pièces, grâce à l'enchaînement de la réplique de la reine Gertrude avec celle de la narratrice de Cendrillon $\ldots »^{8}$.

\section{Father and son}

Nous avons donc commencé par travailler Hamlet, dans un temps assez court (sur six séances de trois heures), avec le projet affirmé d'entrée d'en faire une introduction d'une demi-heure environ à la présentation, plus longue, de Cendrillon. Le texte a été découpé de manière à en resserrer les enjeux autour de la question de l'orphelin et de la parole du mort. Ont été retirés de notre projet tout le début avec les gardes, tout ce qui touche à Laërte, à Claudius. Notre travail s'achève avec la conversation de Hamlet avec sa mère, à la fin de l'acte III.

7 La scène est centrée autour du personnage d'Hamlet, dont on imagine qu'il dort. C'est dans son sommeil que lui apparaissent, comme dans un cauchemar, le fantôme de son père, Polonius, Ophélie, sa mère. Le plateau est tout entier couvert de draps blancs (qui seront les draps du lit d'Hamlet mais aussi les carnets dont il emplit les pages blanches de " words, words, words ", ou encore les nuages qui prennent des formes d'animaux au gré de ses divagations, et la camisole de force dans laquelle on l'emmène finalement). Enroulé dans ses draps, le jeune Hamlet, bercé par des parents aimants, écoute une berceuse. Nous avons choisi de commencer la pièce par la chanson « father and son » de Cat Stevens qui intervient dans Cendrillon: c'est alors le prince qui attend sa mère et chante pour son anniversaire Il est assez étonnant que Joël Pommerat ait choisi cette chanson qui évoque les rapports père-fils (le Roi, père du très jeune prince, est une présence très discrète) alors que toute la pièce tourne autour du manque de la mère... Nous commençons donc Hamlet, dans la langue de Shakespeare, par cette berceuse interprétée en direct sur le plateau par quatre des élèves qui chantent tandis que trois autres les accompagnent à la guitare.

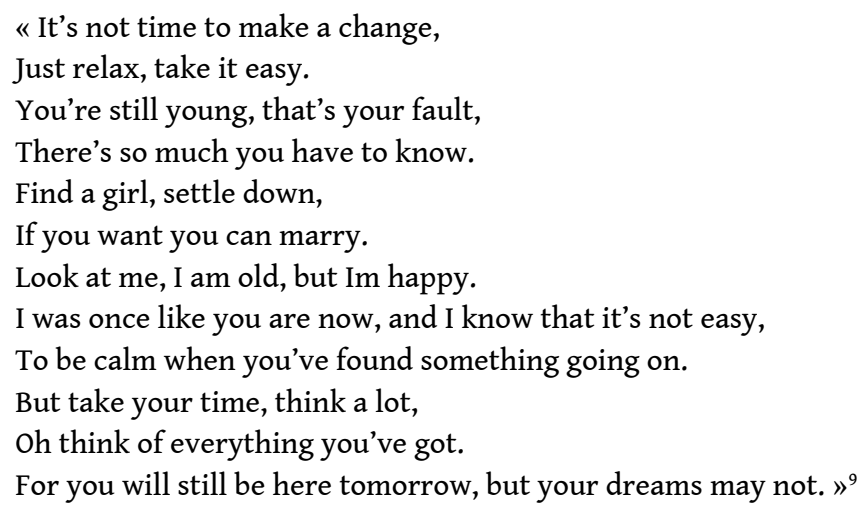

8 A la fin de la berceuse, les accords se font dissonants, les chanteurs s'écroulent dans les draps et le jeune Hamlet se réveille en sursaut. 
Cette scène initiale aura un double écho dans Cendrillon. Le premier a été imaginé très tôt. Comme il est indiqué dans les didascalies de Cendrillon, le jeune Prince chante la chanson de Cat Stevens ${ }^{10}$. Le prince est interprété par un des élèves guitariste qui accompagnait la chanson dans le début d'Hamlet. Il chante maintenant en playback, micro à la main, dans une ambiance de discothèque, ancrant ainsi résolument notre Cendrillon dans l'univers de Pommerat. Il ne s'agit pas de répéter la scène mais d'en proposer une variation qui inscrit ce motif musical dans la réflexion commune aux deux pièces autour du rapport parentenfant. Le deuxième écho est apparu au cours du travail, d'une manière quasiment fortuite. Si nous avons inventé la scénographie du cauchemar pour Hamlet, nous n'avons fait que suivre les indications de Pommerat pour trouver une scène de cauchemar dans Cendrillon. Lors de la première nuit dans sa nouvelle chambre, Sandra a peur et chante pour se donner du courage. Le hasard de la distribution a voulu que l'élève qui interprète ce passage soit celle qui ouvre également Hamlet dans le rôle titre. L'élève a adopté la même posture, enroulée dans les draps. Ces draps sont blancs dans Hamlet et jaunes dans Cendrillon puisque nous avons choisi d'illustrer le passage d'un univers à l'autre par une colorisation du plateau, des costumes et accessoires. Quand, pour se rassurer, Cendrillon se met alors à chanter la comptine du petit Prince qui remet toujours sa visite au lendemain, l'image d'Hamlet, prince de la procrastination, a surgi de manière inattendue, comme si la très jeune fille faisait ici appel à la figure lointaine de ce cousin éloigné mais si proche. Les chansons nous ont donc soutenus dans notre projet et ont confirmé de diverses manières notre intuition de départ.

\section{D'une mère à l'autre : les mots}

10 Dans notre montage, la partie Hamlet s'achève sur la mère, seule, sur un plateau couvert de draps froissés, comme un lit déserté. Tournée vers le public, la mère promet alors à ce fils empoisonné par des paroles insensées : «N'en doute pas : si les mots sont le souffle / et le souffle la vie, jamais ma vie/ Ne soufflera un mot de ce que tu m'as dit $»^{11}$. Gertrude reste sur scène, et commence à ramasser les draps épars qui deviennent un lit dans lequel elle se couchera. Pendant ce temps, derrière le rideau de fond qui sépare notre plateau des coulisses, tout le monde quitte son pyjama blanc pour revêtir les pièces bariolées des costumes de Cendrillon. Arpenté par Gertrude, le plateau n'est pas vide, les lumières restent allumées et la transition d'une pièce à l'autre n'est clairement pas une rupture. Le changement de costume nécessitant un certain temps, nous avons proposé à l'élève qui interprète Gertrude de répéter plusieurs fois cette dernière phrase, comme si elle se la disait à elle-même pour bien faire résonner son mystère, ses zones d'ombre.

11 Entre alors un personnage très coloré, vêtements rouge, jaune et violet, qui ressemble à un des Hamlet blancs que nous venons de quitter. Il termine de s'habiller à vue, sur le plateau. Il s'agit bien sûr du même élève, que Gertrude regarde avec un temps d'hésitation : sommes-nous encore dans Hamlet ou déjà ailleurs? Il prononce alors ce qui sera pour nous les premières paroles du Narrateur «Dans l'histoire que je vais vous raconter, les mots ont failli avoir des conséquences tragiques sur la vie d'une très jeune fille. Les mots sont très utiles mais ils peuvent aussi être très dangereux... ${1^{12}}^{2}$. La reprise du même terme, « les mots ", lié aux idées de vie et de mort, instaure immédiatement le lien entre les deux œuvres. La mère est liée au langage. Si la narratrice de Cendrillon parle avec un accent dans la mise en scène de Pommerat, c'est qu'elle «a oublié la langue que 
ma mère m'a apprise ", comme si grandir c'était trouver ses propres mots, sa propre langue en acceptant de se séparer de sa mère.

Cendrillon peut commencer. Sans quitter le plateau, Gertrude devient donc la mère de Sandra. Et c'est bien la mère de Cendrillon qui est couchée sous nos yeux, affaiblie par la maladie qui va emporter son "souffle", "sa vie " et faire de ces mots un souffle presque inaudible que sa fille, une enfant pleine de vie qui nie la maladie de sa mère et ne lui parle qu'avec un casque (à musique) sur les oreilles, ne pourra entendre. Dans un souffle, la mère de Cendrillon délivre un message de vie qui, mal compris, deviendra mortifère. Les deux mères, interprétées par la même actrice et dans le même costume (une chemise de nuit blanche), dorment dans les mêmes draps et font le lien entre les deux parties de la représentation.

\section{De la mère au père : la question du spectre}

Dans Hamlet, la question du spectre, du père mort, pose toujours problème dans la représentation. Nous avons choisi de faire apparaitre ce père très concrètement sur le plateau, comme les autres personnages qui peuplent l'imaginaire du dormeur. Les draps et les vêtements blancs trouvent ainsi une autre signification : ils disent le fantôme dans son suaire. Trois garçons interprètent ce fantôme dans un rôle collectif. Lors de la première apparition, ils sont tous les trois debout et forment un triangle autour d'Hamlet qui semble ainsi pris dans un étau et ne sait plus où donner de la tête selon que c'est l'un ou l'autre qui parle. Lors de la deuxième apparition, avec Gertrude, le fantôme forme un seul être à trois têtes qui se tient derrière Gertrude et constitue un bloc étrange et improbable sur le plateau.

Dans Cendrillon, Joël Pommerat ne propose pas de véritable spectre. La mère de la très jeune fille meurt. Elle est ensuite absente du plateau. Son esprit hante pourtant la très jeune fille qui ne lâche pas sa montre et son obsédante mélodie "ah vous dirais-je maman ». La robe de la mère qui accompagne Sandra dans son sommeil et que le père déplace maladroitement sur un spectral mannequin constitue à sa manière un fantôme. Avec mes élèves de terminale, nous avons choisi de ne pas garder la robe, ni exactement la montre. En revanche, nous proposons à la mère de rester sur scène après sa mort. Là encore, la proposition est née des contraintes de la présentation : lors de la distribution des rôles, l'élève qui jouait la mère de Sandra aurait aimé incarner aussi la fée, mais, le rôle étant très convoité, nous l'avons attribué à un garçon et à une fille qui n'avaient pas d'autres rôles encore ${ }^{13}$. Au final, l'élève se retrouvait peu servie, limitée à deux apparitions pauvres en texte en début et en fin de pièce. Nous avons donc cherché à lui inventer une présence plus valorisante. Il lui a été proposé de rester sur le plateau, muette et glissante, pieds nus, en lien avec Sandra par le regard. Ce qui n'était au départ qu'un pis-aller est devenu une des lignes de force de notre proposition. Dans sa chemise de nuit blanche qui tranche avec les couleurs des autres personnages, la mère constitue un fantôme familier qui reste aux cotés de sa fille, l'enveloppe de quelques gestes maternels (la recoiffe, l'apaise par quelques gestes lents, ..). A partir de la scène 4 de la première partie, elle porte autour $\mathrm{du}$ cou une grosse montre. L'accessoire et le personnage forment ainsi un tout. Cette incarnation de la morte fait pendant au fantôme paternel d'Hamlet. Dans les deux cas, cette présence raconte comment l'esprit de l'orphelin qui refuse de faire son deuil, d'accepter l'absence, fabrique une résilience du disparu. 
Les deux pièces racontent comment ne pas faire son deuil, c'est avoir peur d'oublier, se sentir responsable du souvenir du mort, coupable de le tuer si on l'oublie. Hamlet et Sandra se sentent assassins en puissance, car oublier c'est faire mourir l'autre. Ils se punissent de ce meurtre qu'ils n'ont pas commis en devenant autre : fou ou souillon. Ils refusent de vivre, car ils croient qu'ils feront alors mourir celui qui leur a donné la vie.

\section{Double Magie}

L'autre difficulté que pose la pièce de Pommerat pour une mise en scène dépourvue d'effets techniques spéciaux sophistiqués est celle des interventions magiques. Si Hamlet pose la question du spectre, Cendrillon interroge celle de la fée. Là encore, c'est au cours du travail de plateau et dans la perspective de notre rapport à Hamlet, que des clés ont été trouvées.

Plusieurs élèves souhaitant s'essayer au rôle de la fée, nous avons choisi de la distribuer à deux d'entre eux, un garçon et une fille. Comme le spectre est triple, la fée sera double. Dans Cendrillon d'ailleurs, la plupart des personnages fonctionnent par deux ${ }^{14}$. Notre projet est lui-même un diptyque. Une double fée donc, ou plutôt une fée gaffeuse et son assistant désespéré, soutiennent le comique de la scène. Tout fonctionne bien pour la première intervention de la fée. Se pose ensuite la question de la métamorphose: comment jouer les improbables transformations de Sandra? Après plusieurs tentatives peu probantes, pour changer de costume en coulisses, n'utiliser qu'un accessoire à chaque fois, etc., nous avons décidé que l'élève jouant Sandra disparaîtrait dans la coulisse pour la première métamorphose. Réapparaît alors l'élève qui a joué la première Sandra... mais elle est vêtue de son costume d'Hamlet (un pyjama noir et blanc avec un bonnet pour le rôle de Rosencrantz)! La fée semble s'être alors trompée de pièce. Le clin d'œil à Hamlet permet à notre Sandra de la deuxième partie de se changer tranquillement en coulisses ! Hamlet nous a aidé là où on ne pensait pas à lui a priori !

18 Enfin, dans la mise en scène de Pommerat, une ultime scène vient donner la clé de l'erreur annoncée dans laquelle la jeune fille s'est fourvoyée. Par la magie de la fée et de la vidéo, Pommerat nous donne à revoir la scène initiale et les paroles mal entendues sont alors prononcées à nouveau: tout s'explique, Sandra avait confondu «mourir » et « sourire » et, au petit jeu des paronymes, a failli gâché sa vie pour une erreur d'audition. Dans notre mise en scène, l'élève qui joue la fée dans la première partie a été affectée à la régie, sons et lumières, pour la deuxième partie. L'association magie et technique se fait donc naturellement. Cependant, pour la scène de la révélation, notre fée-technicienne quitte sa régie et traverse le plateau pour aller chercher en coulisses la première Cendrillon (trois élèves se partagent le rôle) qui rejoue donc pour la dernière Cendrillon la scène du début.

19 En fin de parcours, Hamlet choisi la mort, le sang, tandis que Sandra abandonne le monde $\mathrm{du}$ souvenir pour se lancer dans la vie, la rencontre de l'autre. Nous avons alors abandonné la tragédie élisabéthaine et, toujours en chanson, basculé dans l'univers disco et stroboscopique de la fête populaire : notre présentation de Cendrillon s'achève sur un bal au son d'un tube contemporain de Bruno Mars : «It's a beautiful night, We're looking for something dumb to do. Hey baby, I think I wanna marry you... ». Cohérente avec l'univers pop des spectacles de Joël Pommerat, cette note finale fait un clin d'œil à la formule de clôture des contes « ils se marièrent et eurent beaucoup d'enfants » que le 
texte dément en contrepoint «même après que la vie les a éloignés l'un de l'autre, le prince et la très jeune fille s'écrivirent ${ }^{15}$ ». Cette mystérieuse correspondance entre le Prince et Sandra tisse notre ultime lien intertextuel entre Hamlet et Cendrillon.

Et si à l'issue de la farandole, l'élève qui a joué la mère, toujours en chemise de nuit, vient récupérer le seau abandonné un peu avant par Sandra-Cendrier et se retrouve face à Follavoine ${ }^{16}$ qui s'énerve " Ah ça, tu es folle? tu m'apportes ton seau de toilettes ici à présent ? ... : il est près de onze heures et tu es encore là à traîner en souillon! », ce ne sera qu'une improbable coïncidence permise par un programme plus malicieux qu'on ne serait tenté de le croire!

\section{NOTES}

1. Voir les cinq volumes de la série Ecrivains de plateau publiée aux éditions des Solitaires intempestifs entre 2005-2013.

2. Catherine Vuilliez travaille avec le CDN D'Orléans dirigé par Arthur Nauzyciel sous la direction de qui elle a entre autres joué dans Ordet et dans La Mouette. Notre groupe est constitué de treize élèves.

3. Joël Pommerat, Cendrillon, Arles, Acte Sud, coll. Babel, acte I scène 10, p. 38.

4. William Shakespeare, Hamlet, traduction de Yves Bonnefoy, Paris, Gallimard, coll. Folio, acte I, scène 5.

5. Joël Pommerat, Cendrillon, op. cit., p. 12.

6. Carnet de bord de Noémie, élève de terminale L3.

7. Il aurait été possible aussi de travailler sur les deux couples "siamois » qui dans Hamlet (Rosencrantz et Guildenstern) comme dans Cendrillon (les sœurs, la grande et la petite) s'allient au beau-parent pour surveiller et malmener l'orphelin. Nous n'avons pas choisi de souligner cette ressemblance dans notre proposition scénique afin de ne pas enfermer les élèves dans des schémas trop systématiques.

8. Carnet de bord de Mathilde, élève de terminale L3, exposant le projet d'ensemble.

9. Cat Stevens est mort en octobre 2013, alors que nous étions en train de travailler sur cette berceuse introductive aux deux pièces. Cette disparation a ajouté une perspective fantomatique à notre propos spectral.

10. Joël Pommerat, Cendrillon, op. cit., acte II scène 7, p. 80.

11. Shakespeare, Hamlet, op. cit., acte III scène 4 .

12. Joël Pommerat, Cendrillon, op. cit., acte I scène 1, p. 9.

13. La fée est une magicienne maladroite qui est d'abord accompagnée d'un assistant muet mais très actif. Dans la deuxième apparition, l'assistant intervient seul, l'élève a supplanté le maître comme un enfant qui aurait « tué » son parent...

14. Voir l'article d'Alexandra von Bomhard dans ce dossier.

15. Joël Pommerat, Cendrillon, op. cit., acte II scène 15, p. 112.

16. La troisième partie du programme d'œuvres est constitué de deux pièces de Feydeau : Un fil à la patte et On purge Bébé, dont la réplique de Follavoine est évidemment extraite. 